\title{
Fluid Mechanics of the New Cartesian Physics
}

\section{Dizhechko Boris Semyonowich*}

Lenin prospect 85, Apartment 16, City of Sterlitamak, Bashkortostan, Russia

New Cartesian Physics represents a generalization of the theory of relativity, quantum mechanics and other physical theories based on the principle of identity of space and matter of Rene Descartes. She argues that rotation of matter creates the physical space, which in the state of the physical vacuum is realized by the person as the length and is not perceived by them as a body. To become as a body, particles of space must rotate in a more complicated way than in the physical vacuum. The stability of particles due to known from the theory of relativity by slowing down time, i.e. the achievement of space the speed of light in the center of rotation of the particles, which leads to internal deceleration time until it stops, i.e., to the particle stability.

According to Descartes space is the environment and every movement in it is done in a circle, as no part of them cannot get away from his seat otherwise than in the place of the other part, which in turn goes to the third place, etc. "When a body," he writes, " leaves its place another body, banishing it, it takes the place of the third body, and this is the last - place fourth, and so on until the last which at that moment occupies the place left the first of the bodies." This movement is called the Descartes vortex by analogy with the observed air-eddies and whirlpools, which are also vortex motion of the space being of air or liquid. For New Cartesian Physics body is the accumulation particles of space in a rotational movement, therefore they have the same spatial nature as the physical vacuum. Corporeality and length of it laid in the identity of space and matter. This principle of identity allows us to recognize each point in space the material so that you can associate with it an infinitesimal system of reference in which the maximum speed signal is equal to the speed of light. Moving from one infinitely small frame of reference to another, the light representing the fluctuation of the space, maintains its speed regardless of their motion relative to each other. The fluctuation of the space changes its frequency according to the Doppler Effect, if a motion of the infinitesimal reference systems associated with its points, with respect to each other. Einstein's postulate that all inertial frames the speed of light is the same, is essentially a statement about the identity of space and matter, if the inertial system is considered infinitely small, and associated with points in space (of matter).

In new Cartesian physics is no question about the proper line of motion is straight or circular. Linear motion is represented by motion on a circle of infinitely large radius, i.e., along the trajectory of an infinitesimal curvature. Theory of relativity it was revealed that the rectilinear motion is reduced length and deceleration time, i.e. there is a conversion of one space (resting) to another (moving) and Vice versa. Reducing the infinitely large radius of the circular motion leads to an increase in speed according to the law of conservation of angular momentum, which in turn leads to a further increase in speed, etc. as long as the speed will not be close to the speed of light. Time slows to almost zero and the rotation of the space inside the vortex becomes eternal in relation to the external world. So the vortices become particles, which can be linked into other vortices, molecules, planets, stars. Energy on the formation of vortices and their Union is drawn from the Universe itself and remains as an integral part.

Consider the motion of space along the circumference of an infinitely large radius, i.e. an inertial reference frame. Inertial system, leaving your seat empty, displaces another system, which takes the place of the third system, which takes the place of the fourth, and so on until the last one, which comes to an empty place after the first reference system. Thus, along the circumference passes the wave of perturbation, speed which cannot be more than the speed of light and the last inertial system takes place first than instantaneously, as in Descartes, but after a certain period of time, in this case infinite. This means that straight-line motion no way in space, and it can only move along paths having a finite curvature. The vector of this movement is decomposed into component vectors, one of which is directed in the direction of a hypothetical straight line, and the other radius of curvature. As in the theory of inertial reference system moving uniformly in a straight line, the delay period is maintained during the entire time of motion. From this it follows that the circle does not correspond to a valid trajectory. This leads to the known correction of physical quantities characterizing the process movement. Further, if we decrease the radius of an infinitely large circle, so that it was not such, but was infinitely small circumference, it is obvious that these ratios will continue, and will characterize the microcosm with all its quantum properties.

Further, formulas of classical physics allow the exclusion of these masses, i.e. the values characterizing the physicality of tangible objects and they become formulas characterizing the movement space. So, for example, the formula of the law of conservation of angular momentum when the mass becomes Kepler's laws, describes the motion of a point of space: $v_{1} R_{1}=v_{2} R_{2}$ for equal time intervals the radius vector of the point covers an equal area.

Another example is the movement of points of space around gravitational mass, the speed of which does not depend on body mass in these points:

$$
v=\sqrt{\frac{\gamma M}{R}}
$$

Thus, here the physical space constitutes a field in which the inertial mass in each its point moves around a gravitational mass at a rate that does not depend on the body mass.

If you take the formula of Bernoulli's equation for fluid flow, it also allows the exclusion of the mass of its particles.

$$
\frac{\rho v^{2}}{2}+\rho g h+p=\text { const }
$$

Here

$\rho$ - The density of the liquid,

*Corresponding author: Dizhechko Boris Semyonowich, City of Sterlitamak, Bashkortostan, Russia, Tel: 7 4997041341; E-mail: fizika3000@yandex.ru

Received March 27, 2017; Accepted September 30, 2017; Published October 20, 2017

Citation: Semyonowich DB (2017) Fluid Mechanics of the New Cartesian Physics Fluid Mech Open Acc 4: 179. doi: 10.4172/2476-2296.1000179

Copyright: @ 2017 Semyonowich DB. This is an open-access article distributed under the terms of the Creative Commons Attribution License, which permits unrestricted use, distribution, and reproduction in any medium, provided the original author and source are credited. 
$v$ - The velocity of the flow,

$h$ - Height where there is a considered fluid element,

$p-$ pressure at the point in space where the center of mass of the considered fluid element,

$g$ - The acceleration of free fall.

Express the pressure $p$ force on the Newton's law:

$p=\frac{F}{S}=\frac{m a}{S}=\rho a l$

By substituting the expression $\rho a l$ in Bernoulli's formula, we get:

$$
\frac{\rho v^{2}}{2}+\rho g h+\rho a l=\text { const }
$$

Where $l$ - the average free path length of the particle.

Reducing this expression for the density of the medium $\rho$, we obtain a formula Bernoulli without mass, which characterizes the movement of the points of physical space.

$$
\frac{v^{2}}{2}+g h+a l=\text { const }
$$

If you put $\mathrm{h}=\mathrm{R}$ and divide the expression for this radius, we get the expression:

$$
\frac{v^{2}}{2 R}+g+a \frac{l}{R}=\text { const }
$$

This shows that the sum of half of the centrifugal acceleration, acceleration of free fall and speed up the movement of a point in space is constant.

The examples show that a corpuscle, bodies, planets, stars, etc., which having mass, are reference points indicating the movement of the space. For example, the Earth indicates that the Sun revolves around not just her, but all circumsolar space.

Thus, Fluid Mechanics becomes Space Mechanics of the New Cartesian Physics, which aims at considering the motion of each point in space individually and in combination, to explain the formation and motion of particles, bodies, planets, stars etc. in the Universe. 